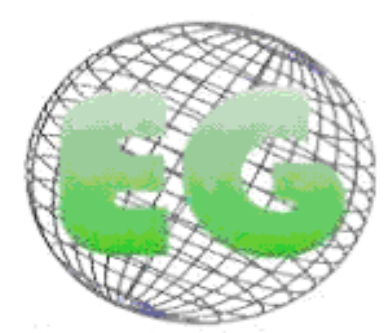

ISSN 1695-6141 N'25

\title{
Funcionalidad y recomendaciones nutricionales de ácidos grasos esenciales y sus derivados en la alimentación del lactante a partir de los 6 meses de edad
}

Functonality and nutritional recommendations of essential fatty acids and their derivatives in breastfeeders of six months onwards

\author{
*Matencio Hilla, E.; Abellán Ballesta, P.; Romero Braquehais, F. \\ *Instituto de Nutrición Infantil Hero, Alcantarilla (Murcia), España. \\ Palabras clave: ácidos grasos esenciales AGEs; ácidos grasos poliinsaturados de cadena larga AGPI-CL; \\ nutrición infantil; alimentación complementaria.
}

Keywords: essential fatty acids; EFAs; long-chain polyunsaturated fatty acids; infant nutrition complementary foods.

\section{RESUMEN}

Los requerimientos de ácidos grasos esenciales (AGEs) y ácidos grasos poliinsaturados de cadena larga (AGPI-CL) durante los últimos meses de gestación y los primeros años de vida son importantes de acuerdo al crecimiento y desarrollo neuronal que está teniendo lugar durante este periodo de vida.

El objetivo de este trabajo ha sido destacar la importancia de garantizar un correcta ingesta de AGEs para cubrir los requerimientos nutricionales de estos, a través de la ingesta de alimentos que contienen en su composición nutricional a estos ácidos de forma natural o han sido enriquecidos con ellos.

La metodología empleada para este fin ha sido la utilización de bases de datos electrónicas como MedLine, ScienceDirect, Scirus, Trip database y de la hemeroteca científica de la Universidad de Murcia; También han sido consultadas las páginas web de organismos nacionales e internacionales tales como la Asociación Española de Pediatría (AEP), Agencia Europea de Seguridad Alimentaria (EFSA) y European Society of Pediatric, Gastroenterology, Health and Nutrition (ESPGHAN).

Los resultados obtenidos muestran como los AGEs e incluso los AGPI-CL (DHA y ARA) son esenciales para el correcto desarrollo del lactante y niño de corta edad; pues aún estando el lactante desde el primer día de vida dotado de todo el sistema enzimático necesario para la conversión de AGEs en sus correspondientes AGPI-CL y sus derivados, esta conversión podría ser insuficiente, siendo necesario y/o esencial el aporte de estos AGPI-CL a través de la dieta.

Las conclusiones derivadas de este trabajo fueron que durante los seis primeros meses de vida el aporte de AGEs y AGPI-CL está asegurado a través de la leche materna o en su defecto a través de las fórmulas de 
inicio; a partir de los 6 meses con la introducción de la alimentación complementaria y la introducción de la leche de vaca a partir de los 12 meses de vida (introducción en contra de las recomendaciones de ESPGHAN), podría verse comprometida la aportación de estos ácidos. Existe un vacío legal en cuanto a la aportación de AGEs a través de la alimentación complementaria distinta a los cereales; sin embargo, comités como el de la AEP recomienda que en los tarritos infantiles de carnes y pescados con verdura, el aporte de AL sea entre 3$4.5 \%$ de la energía de la dieta y al menos $0.5 \%$ desde ALN para satisfacer los requerimientos de AGEs en lactantes y niños de corta edad.

\section{ABSTRACT}

Essential fatty acids and long-chain unsaturated fatty acids are important for growth and neuronal development during the final months of gestation and the early years of life.

The aim of this study is to highlight the importance of ensuring a correct intake of EFAs for nutritional requirements through the intake of foods which naturally contain these acids or which have been enriched with them.

The methodology used was basee on the review of electronic databases MedLine, ScienceDirect, Scirus, Trip database and that of the scientific journal library of the University of Murcia. National and international websites such as the Asociación Española de Pediatría (AEP), European Agency for Food Safety (EAFS) and the European Society of Pediatric, Gastroenterology, Health and Nutrition (ESPGHAN) were also consulted.

The results show how EFAs and also AGPI-CL (DHA and ARA) are essential for the correct development of breastfeeders and infants. Even though the breastfeeder is enzymatically fully equipped from the first day of life to convert EFAs into AGPI-CL and derivatives, the conversion may be insufficient and it may be necessary to include AGPI-CL in the diet.

The conclusions are that during the first 6 months of life the provision of EFAs and AGPI-CL is assured thanks to the mother's milk or through starter formulas; at six months the introduction of complementary foods and the adaptation to cow's milk at twelve months (against the recommendations of the ESPGHAN) may affect the provision of these acids. There is a legal vacuum in the issue of the provision of EFAs through complementary foods other than cereals although committees like the AEP recommend that AL in meat and fish baby foods with vegetables should suppose $3-4.5 \%$ of the energy provision, and ALN at least $0.5 \%$ in order to satisfy EFA requirements in breastfeeders ans infants.

\section{INTRODUCCIÓN}

La garantía en el suministro dietético de los ácidos grasos esenciales (AGEs) es una cuestión de capital importancia, habida cuenta del papel crucial que representan en numerosas funciones y procesos relacionados con el desarrollo del lactante y del niño de corta edad $^{(1,2,3,4)}$. En productos destinados a niños menores de 4-6 meses, este suministro se encuentra garantizado al constituir un requisito legal la composición grasa de las fórmulas infantiles, tanto en la proporción relativa como en la relación ponderal recomendada entre los AGEs ${ }^{(5)}$. Sin embargo, a partir de los 4-6 meses de edad, el suministro podría verse comprometido de no aplicarse pautas correctas en la alimentación del lactante y del niño de corta edad $^{(6)}$. No ha sido establecido el requisito legal para que, especialmente las comidas preparadas que constituyen platos completos, incluyan estos ácidos grasos en su componente graso, bien sea por su contenido natural o por adición de grasas que los contengan. La ingesta de AGEs en las etapas tempranas del desarrollo quedaría potencialmente comprometida si la alimentación no es suficientemente diversificada y equilibrada, especialmente a partir del primer año de vida ${ }^{(1,4,6)}$. Las fórmulas infantiles y las fórmulas de continuación sí garantizan la aportación mínima de estos AGEs, ya que se encuentran estrictamente reguladas en la Unión Europea ${ }^{(5)}$. Pero, a partir del primer año, el niño puede no alimentarse de fórmula de continuación y utilizar la leche de vaca directamente, leche que no contiene los AGEs en proporción y relación adecuadas. 


\section{OBJETIVOS}

Este trabajo pretende resaltar la gran importancia de garantizar la ingesta de estos AGEs en las etapas tempranas del desarrollo. Analizar las posibles formas de conseguir esta garantía y proponer una regulación en este sentido para los preparados completos, con base de carne o de pescado, destinados a niños pequeños a partir de los 4-6 meses de edad.

\section{MATERIAL Y MÉTODOS}

Se han utilizado bases de datos electrónicas como MedLine, ScienceDirect, Scirus, Trip database y de la hemeroteca científica de la Universidad de Murcia. Se han consultado páginas web de organismos científicos como AEP, ESPGHAN, EFSA.

\section{RESULTADOS}

\section{Esencialidad de los Ácidos Linoleico y Linolénico}

Los ácidos grasos linoléico (AL) y y-linolénico ( $A L N)$ son aportados por los alimentos y producen mediante distintos procesos metabólicos, ácidos grasos de cadena larga (AGPI$\mathrm{CL}$ ) que tienen un papel fundamental en el desarrollo de los sistemas visual y nervioso. Los ácidos AL y ALN se consideran esenciales (AGEs) ya que no es posible su formación en mamíferos, al no disponer de los sistemas enzimáticos necesarios para su síntesis. La mayor parte de los AGEs ingeridos con la dieta son utilizados para la obtención de energía por el organismo, solamente una pequeña proporción interviene en el proceso de síntesis de los AGPI-CL. Este proceso tiene lugar mediante reacciones de elongación y desaturación en las que son necesarias diversas enzimas del sistema mitocondrial, cuya actividad es controlada por diversos metabolitos y otros mecanismos (figura 1). Las hormonas, principalmente insulina, y los productos finales del proceso, los propios AGPI-CL, ejercen los efectos más relevantes en estos procesos de síntesis.

Figura 1. Síntesis de AGEs y sus derivados a partir de los precursores

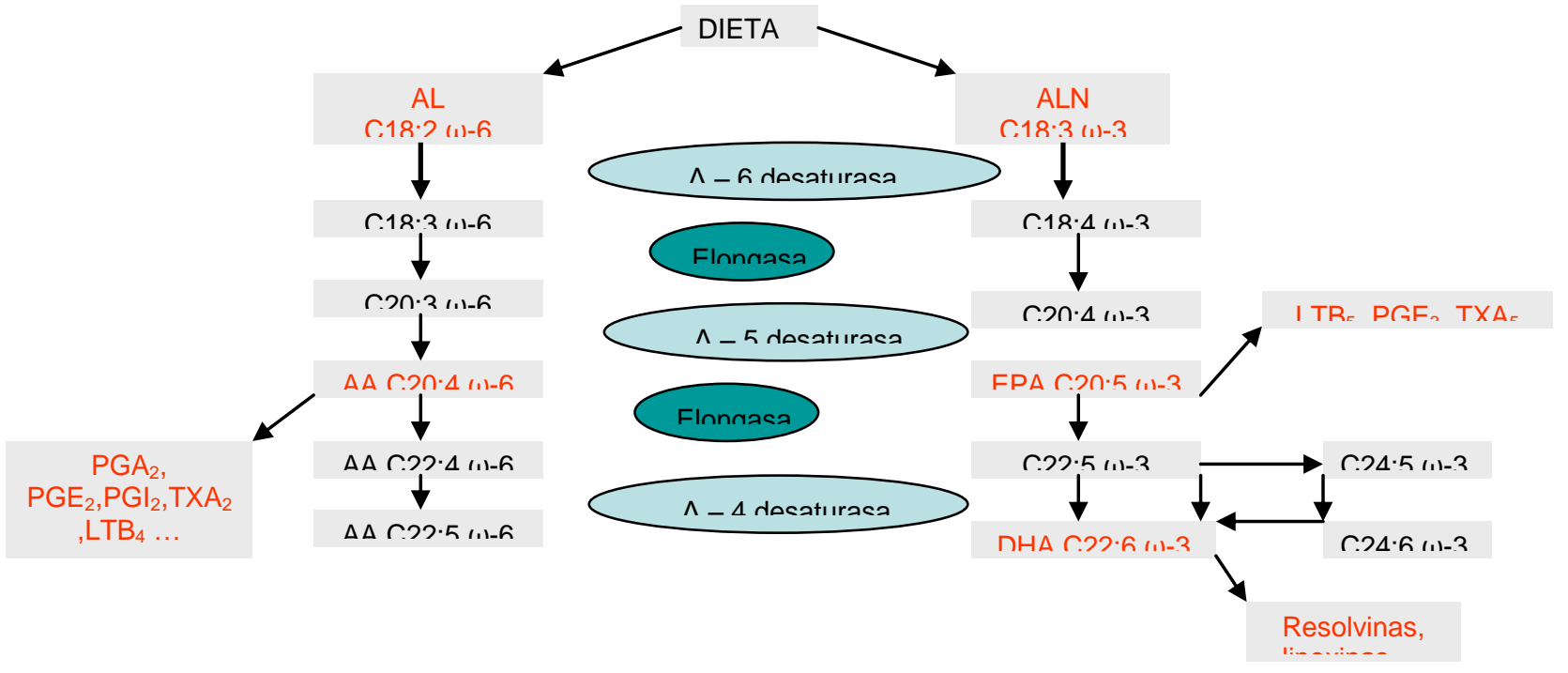

La transformación del $\mathrm{AL}$ en ácido araquidónico (ARA) tiene lugar principalmente en el hígado, de allí es transportado a los tejidos periféricos, formando parte de las lipoproteínas de muy baja densidad (VLDL). Una segunda vía de transporte, es especialmente hacia el 
cerebro, en forma de lisofosfolípido (SN-2 araquidonil fosfatidilcolina) ligado a la albúmina plasmática. El ARA se encuentra en los fosfolípidos y triglicéridos en la posición SN-2. Una vez que es transportado hasta los tejidos, la enzima lipoproteína lipasa vascular, hidroliza a los triglicéridos en las posiciones 1 y 3; el ARA no es liberado por la enzima y la posterior transformación de las VLDL en LDL permite que el ARA quede disponible en los fosfolípidos de las membranas de los tejidos. Posteriormente, la enzima fosfolipasa $A_{2}$ puede liberar el ARA de los fosfolípidos de las membranas, para convertirlo en eicosanoides (prostaglandinas $\left(\mathrm{PGL}_{2}\right)$ y prostaciclinas $\left(\mathrm{PCL}_{2}\right)$ en las células del endotelio; tromboxanos $\left(\mathrm{TX}_{2}\right)$ en las plaquetas; leucotrienos de la series 4 ( $\left.\mathrm{LT}_{4}\right)$ en los leucocitos).

El ALN, al igual que el AL, es transformado en ácido docosahexaenoico (DHA) en el hígado, y se transporta al cerebro, sistema visual y sistema genital, como lisofosfolípido ligado a la albúmina plasmática. En la ruta de biosíntesis de AGPI-CL $\omega-3$ se forma el ácido eicosapentaenocio (EPA), cuya finalidad es la formación de DHA; sin embargo el EPA procedente de la dieta por liberación de lipasa $A_{2}$ y acción de las enzimas ciclooxigenasa y lipooxigenasa da lugar a la formación de eicosanoides $\left(\mathrm{LT}_{5}, \mathrm{TX}_{3}, \mathrm{PCL}_{3}, \mathrm{PG}_{3}\right)$ con efectos antagónicos a los eicosanoides derivados del ARA (figura 1).

La síntesis de AGPI-CL tiene lugar fundamentalmente en el hígado, aunque también pueden ser sintetizados en el sistema nervioso y en el intestino. La retroconversión de C24:6 $\omega-3$ hasta C22:6 $\omega-3$ tiene lugar en los peroxisomas (figura 1). Solamente en el cerebro las mitocondrias además de los peroxisomas, tendrían la capacidad de retroconversión para la síntesis de DHA. La actividad de la enzima delta-6 desaturasa controla la ruta de desaturación-elongación de los AGEs (figura 1). Esta enzima no presenta especificad de sustrato pero sí una mayor afinidad por el sustrato más insaturado, siendo su actividad inhibida por exceso de sustrato o producto (Inhibición de tipo Feed-Back o retroalimentación) (figura 1).

La esencialidad del $A L$ en nutrición infantil fue establecida en un estudio realizado con 428 niños alimentados con una fórmula con leche de vaca y distintos tipos de grasa. En niños alimentados con leche de vaca (con concentraciones trazas de AL y ALN) se observaron descamación y sequedad de la piel, así como retraso del crecimiento ${ }^{(1)}$. El recién nacido y el feto tienen una limitada capacidad de síntesis a partir de los precursores ${ }^{(1,2,3,4)}$. El DHA es aportado por la madre a partir del sintetizado por su organismo, del ingerido en su dieta y de sus reservas. El proceso de neurogénesis finaliza durante las primeras semanas de vida sin embargo, el proceso de sinaptogénesis, en el cual participa activamente el DHA, se prolonga hasta la pubertad. Durante este periodo de tiempo, es de vital importancia un aporte correcto de AGEs desde la dieta, así como alimentos que contengan DHA como fuente natural.

ARA, DHA y EPA junto con otros micronutrientes son cruciales para el desarrollo, integridad y funcionalidad cerebral ${ }^{(3)}$.

\section{Funcionalidad de los AGPI-CL}

El ácido araquidónicio C20:4 $\boldsymbol{\omega}-\mathbf{6}$ (ARA) es un componente de los fosfolípidos de las membranas celulares e interviene activamente en la regulación de estas. A su vez es precursor de eicosanoides de la serie 2, compuestos de 20 átomos de carbono de gran actividad biológica. La síntesis de eicosanoides derivados del ácido araquidónico depende de la disponibilidad de este ácido en los fosfolípidos de las membranas, así como de la actividad de la fosfolipasa $A_{2}$ influenciada a su vez, por una variedad de estímulos hormonales. Los eicosanoides de la serie 2 son muy activos, tienen un tiempo de vida corto 
y actúan sobre las células que lo producen (respuesta autocrina) o en sus proximidades (respuesta paracrina); todas las células del organismo tienen capacidad de síntesis de estos eicosanoides. En procesos inflamatorios agudos, el ARA situado en posición SN-2 en fosfolípidos de las membranas de los leucocitos, es liberado por acción de la fosfolipasa $A_{2}$, convirtiéndose en sustrato para la formación de distintos eicosanoides como protaglandinas, leukotrienos (proinflamatorios) y lipoxinas (anti-inflamatorios) (Tabla I). Los metabolitos del ARA juegan un papel importante tanto en estados iniciales de la inflamación $\left(\mathrm{PG}_{2}, \mathrm{LTB}_{4}\right)$, como en la resolución (lipoxinas) ${ }^{(7,8)}$. Durante la fase inicial de la inflamación, se genera $\mathrm{PGE}_{2}$, implicada en el control del flujo sanguíneo y la vasodilatación; requisitos necesarios para que los leucocitos circundantes lleguen al foco infeccioso, se adhieran y se produzca la diapedesis ${ }^{(7)}$. Los leucotrienos de la serie $4\left(\mathrm{LTB}_{4}\right)$ son potentes quimioatrayentes de neutrófilos y leucocitos en los estados iniciales de la inflamación, mientras que las lipoxinas $\left(\mathrm{LXA}_{4}, \mathrm{LXB}_{4}\right)$ (figura 1), paralizan la infiltración de neutrófilos reduciendo la permeabilidad vascular y la motilidad de las células dendríticas en la resolución de la respuesta inflamatoria $(7,9)$

\begin{tabular}{|c|c|c|}
\hline Entorno de actuación & Eicosanoides & Efecto \\
\hline \multirow[t]{2}{*}{ Sistema cardiovascular } & $\mathrm{PGI}_{2}$ & Efecto hipotensor \\
\hline & $\mathrm{PGF}_{2} \mathrm{LTC}_{4} \mathrm{LTD}_{4} \mathrm{TX}_{2}$ & Vasoconstrictores e Hipertensores \\
\hline \multirow[b]{2}{*}{ Tracto Gastrointestinal } & $\mathrm{PGI}_{2} \mathrm{PGE}_{2}$ & Inhiben la secreción ácida en el estómago \\
\hline & $\mathrm{PGE}_{2}$ & $\begin{array}{l}\text { Estimulan la motilidad intestinal. } \\
\text { Aumentan la secreción de moco en } \\
\text { estómago, intestino delgado. }\end{array}$ \\
\hline Sta. Nervioso Central & $\mathrm{PGI}_{2} \mathrm{PGE}_{2} \mathrm{LTB}_{4}$ & $\begin{array}{l}\text { Pueden amplifican la sensación de dolor en } \\
\text { los procesos inflamatorios. }\end{array}$ \\
\hline \multirow[t]{3}{*}{ Sistema Bronquial } & $\mathrm{LTC}_{4} \mathrm{LTD}_{4}$ & Acción broncoconstrictora \\
\hline & $\mathrm{PGE}_{2}$ & $\begin{array}{l}\text { Relajan la musculatura de bronquios y } \\
\text { traquea }\end{array}$ \\
\hline & $\mathrm{PGF}_{2}$ & Producen broncoespasmo \\
\hline
\end{tabular}

En los últimos años se ha relacionado el aumento de la ingesta de ARA con la incidencia de alergias alimentarias en los países desarrollados ${ }^{(10,12)}$ así como la obesidad infantil ${ }^{(12,13)}$. Se ha estudiado el papel de las lipoxinas en enfermedades como asma ${ }^{(14)}$ y edemas alérgicos (15).

El docosahexaenocio C22:6 w-3 (DHA) es un componente de los fotoreceptores de la retina, participa en la transformación de la señal luminosa en señal eléctrica. Su presencia es importante para mantener la fluidez de las membranas y participa activamente en la neurogénesis, sinaptogénesis y migración neuronal. En las membranas, el DHA no esterificado confiere un grado de flexibilidad e interacción con las proteínas de las membranas, influyendo por tanto en la velocidad de transducción de señales y neurotransmisión ${ }^{(16)}$. En el cerebro la síntesis de DHA es baja o ausente, por lo que la principal fuente para el cerebro es el DHA de la dieta o el sintetizado en el hígado y transportado hasta el cerebro ${ }^{(17)}$. DHA juega un papel importante en la regulación de la expresión génica, en la actividad de los canales de iones y puede ser metabolizado hasta metabolitos neuroprotectores en el cerebro ${ }^{(7,18)}$. La relación entre el contenido en DHA en el 
membranas de los eritrocitos y la agudeza visual ha sido demostrado en numerosos estudios científicos.

El DHA de la dieta, permitirá en estados de respuesta inflamatoria la formación protectinas y resolvinas derivadas de DHA (RvD) y EPA (RvE) en la resolución de la respuesta inflamatoria, reduciendo el tráfico de neutrófilos, regulando la producción de citokinas y especies de oxígeno reactivas, y disminuyendo la magnitud de la respuesta inflamatoria ${ }^{(7)}$. Varios estudios han sugerido que una temprana intervención con ácidos grasos poliinsaturados de cadena larga $\omega-3$ en la dieta de lactantes y niños de corta edad, podría influir la funcionalidad inmunológica y el fenotipo de citokinas durante el desarrollo ${ }^{(10)}$.

El ácido eicosapentaenoico C20:5 w-3 (EPA) de lugar a una serie de eicosanoides de la serie 3, con un comportamiento antagónico a los originados por ARA. Los eicosanoides procedentes de EPA se forman normalmente a partir de dietas en las que se ha administrado EPA como tal, pues el EPA formado en la ruta de desaturación-elongación a partir de ALN ingerido en la dieta, es destinado prácticamente en su totalidad a la obtención de $\mathrm{DHA}^{(19)}$. Desde el EPA procedente de la dieta, se originan en los leucocitos resolvinas ${ }^{(7)}$.

\section{Requerimientos Nutricionales de AGEs y AGPI-CL en lactantes y niños de corta edad.}

Durante la última etapa de gestación tiene lugar una acusada neurogénesis y sinaptogénesis; ambos procesos requieren la presencia de DHA y ARA. Durante este periodo el feto cubre sus requerimientos de DHA y ARA por transferencia placentaria, biosíntesis en el sistema nervioso central y los AG sintetizados en el intestino y el hígado del niño ${ }^{(20)}$. La capacidad de conversión de AGEs en AGPI-CL en el feto no es muy grande. El feto necesita un aporte por la placenta y posteriormente de la leche materna (21). La formación de AGPI-CL $\omega-3$ y $\omega-6$ a partir de sus precursores tiene lugar desde las primeras etapas de vida fetal. Estudios utilizando AL y ALN marcados como isótopos mostraron la capacidad de síntesis de manera endógena DHA y ARA siendo esta síntesis mayor en edades gestacionales tempranas ${ }^{(22)}$.

Algunos estudios sostienen la necesidad de ingesta de $A L$ y $A L N$ por la madre gestante 0 incluso defienden el aporte dietético de DHA desde el consumo de pescado o suplementos de manera que la madre gestante cubra los requerimientos de DHA propios y del feto ${ }^{(3,23,24,}$ 25, 26). El estado de gestación conduce a la madre a un estado de depleción en los niveles de DHA plasmático; la madre tras el parto necesita unos 5-6 meses para recuperar los niveles de DHA en plasma ${ }^{(3)}$. Las últimas recomendaciones emitidas sugieren que la madre en estado gestacional y de lactancia debería ingerir diariamente al menos $200 \mathrm{mg}$ de DHA (23)

Los recién nacidos pretérmino, al contrario de lo que se pensaba hasta hace pocos años, tienen capacidad para sintetizar AGPI-CL a partir de sus precursores ${ }^{(27)}$, aunque de no añadirse ARA y DHA a las fórmulas destinadas a niños nacidos pretérmino, esta capacidad podría ser insuficiente para cubrir sus necesidades ${ }^{(28)}$. Las recomendaciones actuales indican que estas fórmulas deberían contener al menos un $0,35 \%$ del total de ácidos grasos como DHA y un $0,4 \%$ como ARA $^{(29)}$.

El niño nacido a término dispone del sistema enzimático necesario para la transformación de los AGEs en LC-PUFAS, sin embargo hoy en día se desconoce si el AL y ALN pueden ser suficientemente transformados a AA y DHA ${ }^{(30)}$. Todas las elongasas y desaturasas necesarias para la conversión de ALN en DHA son activadas en la primera semana después del nacimiento, aunque las cantidades de DHA producidas a partir de ALN pueden ser 
inadecuadas para soportar los niveles de DHA observados en niños alimentados a pecho (31)

En el Anexo I y II de la Directiva 2006/141/CE relativa a los preparados para lactantes y preparados de continuación, quedan establecidos los valores mínimo y máximo de ácido linoleico (300-1200 mg/100 Kcal) y de linolénico (mínimo 50 mg/100 Kcal) y la relación LA/LNA entre 5-15. El valor mínimo de AL viene marcado por los requerimientos, mientras que el máximo por la inhibición de las enzimas y la posible oxidación por los peróxidos.

En numerosas publicaciones se ha puesto de manifiesto la relación entre la adición de AGPI-CL (DHA y ARA) en las fórmulas infantiles y el aumento de estos en las membranas de los eritrocitos (parámetro que refleja los niveles de AGs de la dieta), así como una cierta mejora en la agudeza visual y el desarrollo cognitivo ${ }^{(32,33,34)}$. Sin embargo, otros estudios sostienen que la adicción de los precursores en proporciones adecuadas es suficiente para un correcto desarrollo visual y cognitivo, no siendo necesaria la adición de AGPI-CL y no existiendo mejora en la agudeza visual o cognoscitiva a largo plazo ${ }^{(35,36,37,38)}$. No es útil utilizar mayores cantidades de ALN en las fórmulas para aumentar el contenido de DHA en plasma; el aporte debe ser de DHA ${ }^{(39,40,41)}$. Al existir evidencias de que la adición de AGPI$\mathrm{CL}$ en las fórmulas infantiles es segura y presentan ventajas al menos transitorias en la agudeza visual y desarrollo cognitivo, se permite su utilización en las fórmulas infantiles y de continuación ${ }^{(42)}$. La Directiva 2006/141/CE y el Real Decreto 867/2008 cita textualmente:

"Podrán añadirse ácidos grasos poliinsaturados de cadena larga (20 y 22 átomos de carbono) (PCL). En tal caso, su contenido no será superior a:

- $1 \%$ del contenido total en materia grasa para los PCL $w-3$

- $2 \%$ del contenido total en materia grasa para los PCL w-6 [1 \% del contenido total en materia grasa para el ácido araquidónico (20:4 w-6)].

El contenido en ácido eicosapentanoico (20:5 w-3) no será superior al contenido en ácido docosahexanoico (22:6w-3). El contenido en ácido docosahexanoico (22:6 w-3) no excederá del de los PCL $w$-6."

A partir de los 6 meses de edad, los lactantes comienzan a ser alimentados con dietas semisólidas siendo más probable una ingesta reducida de DHA desde la dieta como se refleja en la disminución de los niveles de DHA en el plasma ${ }^{(43,44)}$. Con la introducción de una dieta diversificada y la reducción del consumo de leche materna, los requerimientos de AGEs para el correcto desarrollo del niño serán solventados de acuerdo a su contenido en los homogeneizados infantiles, las fórmulas de continuación y los alimentos elaborados a base de cereales. Es de vital importancia, la correcta elección de las fuentes de grasa (pescados, carnes, aceites vegetales) en los homogeneizados infantiles, así como un diseño del perfil graso de acuerdo a las necesidades energéticas y nutritivas del lactante en esta etapa de crecimiento. La alimentación del lactante y el niño de corta edad con menús donde el aporte de AL y ALN mantenga una correcta relación $\omega-6 / \omega-3$, puede ser decisivo en el correcto funcionamiento de las rutas de desaturación-elongación, con formación de DHA y ARA en proporciones adecuadas para una correcta regulación de las membranas, establecimiento de sinapsis y el desarrollo de agudeza visual. Un estudio reciente mostró como la adicción de DHA en los homogeneizados infantiles guarda una cierta correlación con el contenido de DHA encontrado en las membranas de los eritrocitos y mejora de la agudeza visual medida a los 9 meses y a los 12 meses ${ }^{(34)}$ poniendo de manifiesto un posible beneficio en la suplementación con DHA en los menús dirigidos a la alimentación de lactantes y niños de corta edad. Puesto que la conversión de ALN en DHA en niños de corta 
edad y lactantes es limitada $(<0.2 \%)$, el DHA debería ser introducido en los alimentos tras el destete, en forma de aceites de pescado, pescado o incluso en forma de suplementos dietéticos conteniendo $\mathrm{DHA}^{(3)}$. En el periodo de la alimentación complementaria la ingesta de DHA es variable, por lo que requerimientos de DHA a través de la alimentación complementaria deberían ser definidos ${ }^{(45)}$.

La proporción adecuada AL/ALN para que tenga lugar la formación de DHA y ARA es 5:1 o 10:1. Con un $2 \%$ de energía en forma de ALN se podría inhibir completamente la formación de AGPI-CL $\omega-6^{(20)}$. Cuando en la dieta se aporta una alta proporción de ALN, la formación de derivados del AL se ve comprometida. También ocurre al contrario, por lo que la relación entre estos dos ácidos grasos proporcionados por la dieta se ha de controlar dentro de unos límites bien definidos. En situaciones de déficit de DHA, o ingesta desproporcionada de $A L$ y ausencia de ALN, tiene lugar la formación de ácido docosapentaenoico DPA C22:5 $\omega-6^{(1)}$ reemplazando este ácido al DHA en las membranas de las células del tejido nervioso. Una ingesta excesiva de AL aportada por aceites vegetales (de girasol, de maíz) puede inhibir la formación de DHA a partir de ALN debido a la inhibición de la enzima $\Delta-6$ desaturasa por exceso de sustrato ${ }^{(1,4)}$. La afinidad de la desaturasa-6 por el C18:1 $\omega-9, A O$, es insignificante. La formación en el organismo de derivados de $\mathrm{AO}$ se producirá en estados carenciales de $A L$ y $A L N{ }^{(22)}$. La presencia de $C 20: 3 \omega-9$ en las membranas de los eritrocitos es un indicador de malnutrición así como una relación 20:3 $\omega-9 / 20: 4 \omega-6$, Índice trienotetraeno, superior a 0.2 .

Estudios en humanos han puesto de manifiesto como dietas deficitarias en AL y ALN o deficitarias en ALN, producen una disminución de ARA y DHA en los fosfolípidos del cerebro y un aumento de $\omega-7$ y $\omega-9$ mono y poliinsaturados. Recientemente se ha observado como en las dietas occidentales ricas en $A L$, tiene lugar una formación marginal de los derivados del ALN. Diversas estudios experimentales y datos epidemiológicos desde estudios con lactantes, muestran como LC-PUFAs w- 6 son potentes promotores de adipogénesis llegando a proponerse que los cambios producidos de manera desapercibida en la composición de los ácidos grasos de la fracción grasa ingerida en las últimas décadas, hayan sido determinantes en el aumento de prevalencia del sobrepeso y obesidad infantil (13). Por otra parte, el aumento del consumo de AL y ARA en los países desarrollados, también parece tener cierta relación con el aumento de la prevalencia de alergias. ARA y sus metabolitos $\left(\mathrm{PGE}_{2}\right)$ pueden limitar la respuesta inmune hacia una respuesta proalérgica Th2, aumentando la producción de IL-4, IL-5, promoviendo la síntesis de lgE por las células $\mathrm{B}$ y disminuyendo IL-2 e IFN-y citocinas proinflamatorias ${ }^{(10)}$.

No existe actualmente ninguna directiva o reglamento relacionado con la alimentación complementaria para lactantes y niños de corta edad, donde sean establecidos unos límites al contenido de AGEs en alimentos infantiles, "distintos a los alimentos elaborados a base de cereales". Sin embargo, ya se ha mencionado la importancia de mantener un balance adecuado en la ingesta de estos ácidos debido a la afinidad de la enzima $\Delta-6$ desaturasa y la necesidad de estos ácidos grasos esenciales y sus derivados para el correcto desarrollo cognitivo y crecimiento del niño.

El Comité de Nutrición de la Agencia Española de Pediatría AEP, recomienda que el ácido linoleico aporte entre un 3-4.5\% de la energía de la dieta y el ALN al menos un $0.5 \%$ de la energía para satisfacer los requerimientos de ácidos grasos esenciales, con una relación $\omega$ 6/w-3 comprendida entre 5-15 ${ }^{(6)}$ (ver Tabla II). 


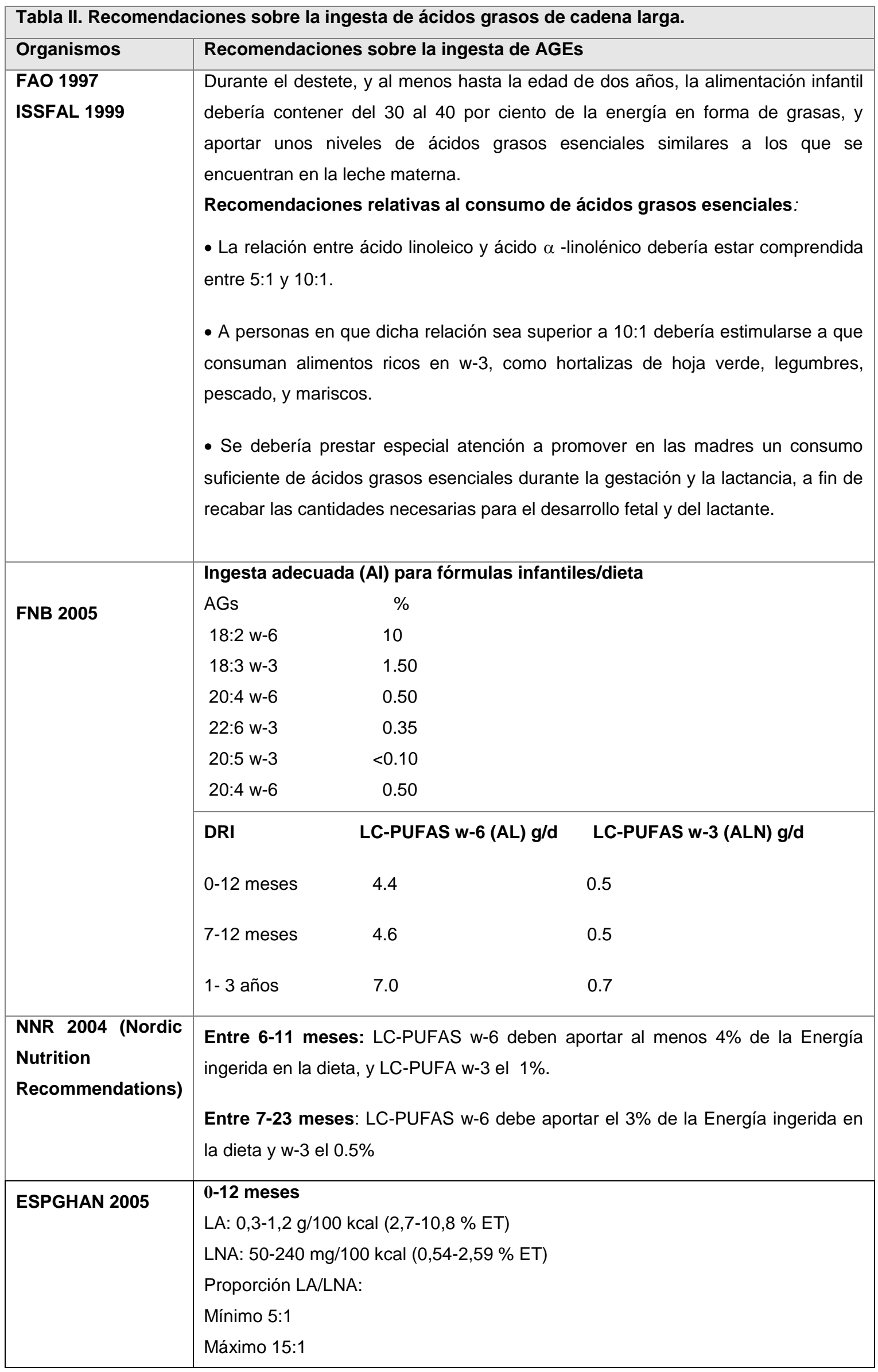




\begin{tabular}{|c|c|}
\hline Organismos & Recomendaciones sobre la ingesta de AGEs \\
\hline $\begin{array}{l}\text { AEP } 2006 \\
\text { Agencia Española } \\
\text { de Pediatría }\end{array}$ & $\begin{array}{l}\text { Ingesta de grasa expresada como \% de la Energía de la dieta: } \\
\text { - } \quad \text { Al menos el } 3-4.5 \% \text { de la E de la dieta como AL y } 0.5 \% \text { ALN } \\
\text { - } \quad \text { Ingesta de AL y LC-PUFAS w- } 6<10 \% \text { de la E de la dieta } \\
\text { - } \quad \text { Poliinsaturados totales }<15 \% \text { de la E de la dieta } \\
\text { - } \quad \text { AG saturados }<10 \% \text { de la E } \\
\text { Utilizar aceite de oliva. (Conferencia de Consenso sobre Lípidos en Pediatría }\end{array}$ \\
\hline $\begin{array}{l}\text { Koletzko et al., } \\
2008 \\
\text { (World Association } \\
\text { of Perinatal } \\
\text { Medicine; Early } \\
\text { Nutrition Academy } \\
\text { and Child Health } \\
\text { Foundation ) }\end{array}$ & $\begin{array}{l}\text { - DHA debería alcanzar al menos el } 0.2 \% \text { y no exceder el } 0.5 \% \text { de los ácidos } \\
\text { grasos. } \\
\text { - Los niveles de ARA adicionados deben ser al menos iguales a los de DHA. } \\
\text { - La cantidad de EPA adicionado no debe exceder la cantidad de DHA. } \\
\text { Parece aconsejable continuar con la provisión de LC-PUFA en la dieta de } \\
\text { niños de más de } 6 \text { meses de edad (mediante adicción en fórmulas de } \\
\text { continuación y alimentación complementaria), aunque las cantidades óptimas } \\
\text { no pueden ser especificadas hasta el momento. }\end{array}$ \\
\hline EFSA 2010 & $\begin{array}{l}\text { - Propone una ingesta adecuada (AI) de } 100 \mathrm{mg} \text { de DHA para lactantes } \\
\text { mayores de } 6 \text { meses y menores de } 24 \text { meses. } \\
\text { - No establece ninguna Al de EPA y DHA para las edades comprendidas entre } \\
2 \text { y } 18 \text { años. Sin embargo aconseja una o dos comidas de pescado ( } 250 \mathrm{mg} \\
\text { de EPA+DHA) a la semana. }\end{array}$ \\
\hline
\end{tabular}

A través de homogeneizados infantiles con perfiles grasos adaptados a las necesidades energéticas y nutricionales de los lactantes y niños de corta edad, de acuerdo a las recomendaciones establecidas por distintos organismos científicos internacionales (ESPGHAN, AAP, FNB), se contribuye al correcto crecimiento y desarrollo neuronal del niño y a la prevención del desarrollo de obesidad o sobrepeso, convertida en los últimos años en la epidemia del siglo XXI.

\section{Fuentes alimenticias de AGEs y sus derivados}

Los aceites vegetales (aceite de girasol, aceite de maiz, aceite sésamo) son la principal fuente de AL, siendo aceite de oliva, aceite de soja, aceite de canola fuentes de ALN. Los aceites vegetales aportan mayoritariamente acido oleico (AO) y $\mathrm{AL}$ y pequeñas cantidades de ALN. Concretamente el aceite de girasol presenta un alto contenido en AL y muy bajo en 
ALN, mientras que el aceite de oliva contiene una cantidad elevada de AO (77\%), y cantidades de $A L(8 \%)$ y ALN (1\%). La soja contiene un $54 \%$ en forma de ácidos grasos w- 6 , $7 \%$ en forma de AGs $\omega-3$ y $24 \%$ en forma de $\omega-9$. Las principales fuentes de LC-PUFAs $\omega-3$ son los frutos secos, vegetales de hoja verde, pescados de agua fría (salmón, sardina, caballa), aceites de pescado.

En la fabricación de fórmulas infantiles, de homogeneizados infantiles destinados a lactantes así como menús dirigidos a niños de corta edad, como fuente de AL y ALN son utilizados aceites vegetales. En las fórmulas infantiles, las fuentes de DHA utilizadas hoy en día son, aceites de pescado, aceites de pescado enriquecidos en DHA, aceites obtenidos de algas o a partir de los fosfolípidos de huevo. Como fuentes de ARA se utilizan los fosfolípidos de huevo o procedente de hongos. Un estudio reciente ha puesto de manifiesto cómo la fuente de DHA y ARA (bien en forma de fosfolípidos de huevo, triglicéridos sintetizados a partir de algas o microorganismos fúngicos) no afecta la biodisponibilidad de este ácido en el organismo del lactante ${ }^{(46)}$. En fórmulas infantiles suplementadas con LC-PUFA se suele emplear un aceite de pescado como fuente de LC-PUFA $\omega$-3 en combinación con otra fuente que proporcione ARA.

Puesto que la presencia de LC-PUFA w-6 es abundante en las dietas que contienen grasas de origen animal, y la presencia de DHA y EPA es más escasa, hoy en día se investiga la posibilidad de obtener DHA a partir de algas transgénicas ${ }^{(47)}$, de plantas transgénicas como fuente de aceite de pescado ${ }^{(48)}$ de la alimentación de gallinas con dietas ricas en ALN, alimentación de cerdos con aceites marinos permitiendo aumentar el contenido de DHA en la carne, incluso se están llevando a cabo experimentos con modificación genética (incorporación del gen $\omega$-3 desaturasa procedente de un gusano invertebrado en ratas ${ }^{(49)}$, para la obtención de nuevas fuentes de DHA como ingrediente funcional.

\section{CONCLUSIONES}

Las siguientes conclusiones son formuladas de acuerdo a las actuales evidencias científicas y recomendaciones:

$\sqrt{ }$ La esencialidad de los AGEs recae en la ausencia en el organismo humano del sistema enzimático necesario para su síntesis, aunque muchos autores consideran a los derivados de estos AGEs (DHA y ARA) como los verdaderamente esenciales dadas las funciones que desarrollan.

$\sqrt{ }$ La proporción y el ratio de AGEs ingeridos en la dieta son determinantes en las vías de desaturación y elongación, pudiendo existir inhibición en la formación de AGPI-CL $\omega-6$ y/o $\omega-3$ por sustrato, producto $u$ otras sustancias que influyen en la actividad de la enzima $\Delta-6$ desaturasa.

$\sqrt{ }$ El recién nacido a término y pretérmino presentan una capacidad limitada de síntesis de AGPI-CL. La leche materna proporciona ARA y DHA al lactante, luego en su defecto las fórmulas infantiles deberían proporcionar al lactante ARA y DHA para su correcto crecimiento, desarrollo cognitivo y agudeza visual.

$\sqrt{ }$ La legislación de fórmulas infantiles y de continuación establece límites mínimos y máximo en el contenido de AL y ALN así como de AGPI-CL. La Directiva Europea sobre alimentos elaborados a base de cereales y alimentos destinados a lactantes y niños de corta edad, no establece límites en los contenidos en AL y ALN en los alimentos distintos de los cereales. 
$\sqrt{ }$ El Comité de Nutrición de la Asociación Española de Pediatría recomienda un aporte de $A L$ entre $3-4.5 \%$ de la energía de la dieta y al menos $0.5 \%$ desde ALN para satisfacer los requerimientos de AGEs y niños de corta edad.

$\sqrt{ }$ La elaboración de alimentos destinados a la alimentación del lactante y niños de corta edad debe realizarse teniendo en cuenta los límites establecidos en la Directiva, y utilizando como fuente de AGEs aceites vegetales que aporten los AGEs en las proporciones recomendadas por los distintos organismos internacionales, de manera que la síntesis endógena de AGPI-CL no sea inhibida o alterada.

\section{REFERENCIAS BIBLIOGRÁFICAS}

1. Uauy R y Castillo C (2003). Lipid Requirements of Infants: Implications for Nutrient Composition of Fortified Complementary Foods. Nutr. 133: 2962S-2972S

2. Díaz-Argüelles Ramírez-Corría V (2001). Suplementación enteral con ácidos grasos esenciales en recién nacidos pretérmino. Rev Cubana Pediatr 73(1):34-42

3. Singh M (2005). Essential Fatty Acids, DHA and Human Brain. Indian J Pediatr 72 (3):239-242.

4. Uauy R and Dangour AD (2009). Fat and Fatty Acid Requirements and Recommendations for Infants of 0-2 Years and Children of 2-18 Years. Ann Nutr Metab 55:76-96

5. DIRECTIVA 2006/141/CE DE LA COMISIÓN de 22 de diciembre de 2006 relativa a los preparados para lactantes y preparados de continuación y por la que se modifica la Directiva 1999/21/CE.

6. Gil Hernández A, Uauy Dagachb R, Dalmau Serrac J y Comité de Nutrición de la AEP (2006). Bases para una alimentación complementaria adecuada de los lactantes y los niños de corta edad. An Pediatr (Barc) 65(5):481-95

7. Serhan CN (2007). Resolution Phase of Inflammation: Novel Endogenous AntiInflammatory and Proresolving Lipid Mediators and Pathways. Annu. Rev. Immunol. 25:101-37

8. Fristche K (2006). Fatty acids as modulators of the immune response. Annu. Rev. Nutr. 26:45-73

9. Aliberti J, Hieny S, Reis e Sousa C, Serhan CN, Sher A. (2002). Lipoxiw-mediated inhibition of IL-12 production by DCs: a mechanism for regulation of microbial immunity. Nat. Immunol. 3:76-82

10. Gottrand F (2008).Long-Chain Polyunsaturated Fatty Acids Influence the Immune System of Infants. J. Nutr. 138: 1807S-1812S.

11. Calder PC, Krauss-Etschmann S, de Jong EC, Dupont C, Frick JF, Frokiaer H et al (2006). Early nutrition and immunity - progress and perspectives. Br J Nutr 96:774-790

12. Massiera F, Saint-Marc P, Seydoux J, Murata T, Kobayashi T, Narumiya S, Guesnet P, Amri EZ, Negrel R, Ailhaud G. (2003). Arachidonic acid and prostacyclin signaling promote adipose tissue development: a human health concern? J Lipid Res. 44(2):271-9

13. Ailhaud G, Guesnet $P$ and Cunnane SC (2008). An emerging risk factor for obesity: does disequilibrium of polyunsaturated fatty acid metabolism contribute to excessive adipose tissue development? British Journal of Nutrition100, 461-470

14. Levy BD, De Sanctis GT, Devchand PR, Kim E, Ackerman K, et al. (2002). Multipronged inhibition of airway hyper-responsiveness and inflammation by lipoxin A4. Nat. Med. 8:1018-2

15. Bandeira-Melo C, Serra MF, Diaz BL, Cordeiro RSB, Silva PMR, et al. (2000) Cyclooxygenase-2-derived prostaglandin E2 and lipoxin A4 accelerate resolution of allergic edema in Angiostrongylus costaricensis-infected rats: relationship with concurrent eosinophilia. J. Immunol. 164:1029-36 
16. Innis Sheila M (2007). Dietary (w-3) Fatty Acids and Brain Development.. J Nutr 137:855859

17. Innis SM (2009). Omega-3 Fatty Acids and Neural Development to 2 Years of Age: Do We Know Enough for Dietary Recommendations? JPGN 48:S16-S24

18. Bazan NG (2006). Cell survival matters: Docosahexaenoic acids signaling, Nereoprotection and photoreceptors. Trends Neurosci 29:263-71.

19. Valenzuela A y Uauy $R$ (2005). Funciones Biológicas y metabolismo de los ácidos grasos esenciales y de sus derivados activos. Tratado de Nutrición, Tomo I, cap 1.13 pág. 429450.

20. Valenzuela A y Nieto S (2001). Acido docosahexaenoico (DHA) en el desarrollo fetal y en la nutrición materno-infantil. Rev Méd Chile 129(10)

21. Rodríguez-Cruz M, Tovar AR, del Prado M, Torres N (2005). Mecanismos moleculares de acción de los ácidos grasos poliinsaturados y sus beneficios en la salud. Rev Invest Clin 57 (3): 457-472

22. Uauy R, Mena P, Wegher B, Nieto S and Salem N (2000). Long chain polyunsaturated fatty acid formation in neonates: Effect of gestational age and intrauterine growth. Pediatr Res 47:127-135

23. Koletzko B, Lien E, Agostoni C, Böhles H, Campoy C, Cetin I et al (2008).The roles of long-chain polyunsaturated fatty acids in pregnancy, lactation and infancy: review of current knowledge and consensus recommendations. J Perinat. Med. 36: 5-14.

24. Jensen C, Voigt R, Prager T, Zou Y, Fraley J, Rozelle $J$ et al. Effects of maternal docosahexaenoic acid intake on visual function and neurodevelopment in breastfed term infants. Am J Clin Nutr 2005; 82:125-32.

25. Lauritzen L, Hoppe C, Maries Straarup L y Michalsen KF (2005). Maternal Fish Oil Supplementation in Lactation and Growth during the First 2.5 Years of Life. Pediatr Res 58: 235-242.

26. Helland IB, Saugstad OD, Saarem K, Van Houwelingen AC, Nylander G, Drevon CA (2006). Supplementation of $w-3$ fatty acids during pregnancy and lactation reduces maternal plasma lipid levels and provides DHA to the infants. J Matern Fetal Neonatal Med. 19(7):397-406

27. Moreno Villaresa JM, Galiano Segovia MJ (2006). Recientes avances en fórmulas infantiles Rev Pediatr Aten Primaria 8 (1):S37-49

28. Qi K, May M, Deckelbaum RJ (2002). Long-chain polyunsaturated fatty acid accretion in brain. Curr Opin Nutr Metab Care. 5:133-138

29. Koletzko B, Agostoni C, Carlson SE, Clandinin T, Hornstra G, Neuringer M et al. (2001) Long chain polyunsaturated fatty acids (LC-PUFA) and perinatal development. Acta Paediatr 90: 460-4

30. Gibson R and Makrides M (2000). w-3 Polyunsaturated fatty acid requirement of term infants. Am J Clin Nutr 71(suppl):25, 1S-5S.

31. Salem N, Brent Wegher JR, Mena P y Uauy R (1996). Arachidonic and docosahexaenoic acids are biosynthesized from their 18-carbon precursors in human infants. Proc. Natl. Acad. Sci. BiochemistryUSA 93:49-54

32. Birch E, Hoffman R, Castañeda Y, Fawcett L, Birch G and Uauy R (2002). A randomized controlled trial of long-chain polyunsaturated fatty acid supplementation of formula in term infants after weaning at 6 wk of age. Am J Clin Nutr 75:570-80

33. Birch E, Castañeda Y, Wheaton D, Birch D, Uauy R and Hoffman D (2005). Visual maturation of term infants fed long-chain polyunsaturated fatty acid-supplemented or control formula for 12 mo. Am J Clin Nutr81: 871-9. 
34. Hoffman D, Theuer R, Castaneda Y, Wheaton D, Bosworth R, O'Connor A, Morale S et al (2004). Maturation of visual acuity is accelerated in breast-fed term infants fed baby food containing DHA-enriched egg yolk. J Nutr 134(9):2307-13

35. Udell T, Gibson RA, Makrides M (2005). PUFA Study Group. The effect of alpha-linolenic acid and linoleic acid on the growth and development of formula-fed infants: a systematic review and meta-analysis of randomized controlled trials. Lipids. 40(1):1-11

36. Auestad N, Scott DT, Janowsky JS, Jacobsen C, Carroll RE, Montalto MB, et al (2003). Visual, cognitive, and language assessments at 39 months: a follow-up study of children fed formulas containing long-chain polyunsaturated fatty acids to 1 year of age. Pediatrics. 112(3 Pt 1):e177-83

37. Ghys A, Bakker E, Hornstra G, van den Hout M (2002). Red blood cell and plasma phospholipid arachidonic and docosahexaenoic acid levels at birth and cognitive development at 4 years of age. Early Hum Dev. 69(1-2):83-90

38. Makrides M, Neumann MA, Jeffrey B, Lien EL, Gibson RA (2000). A randomized trial of different ratios of linoleic to alpha-linolenic acid in the diet of term infants: effects on visual function and growth. Am J Clin Nutr. 71(1):120-9

39. Arterburn LM, Hall EB, Oken H.(2006) Distribution, interconversion, and dose response of w-3 fatty acids in humans. Am J Clin Nutr. 83(6):1467S-1476S

40. Bowen RA, Clandinin MT (2005). Maternal dietary $22: 6 \mathrm{w}-3$ is more effective than 18 : $3 w-3$ in increasing the $22: 6 w-3$ content in phospholipids of glial cells from neonatal rat brain. Br J Nutr. 93(5):601-11

41. Marin MC, Rey GE, Pedersoli LC, Rodrigo MA, Alaniz MJ (2000). Dietary long-chain fatty acids and visual response in malnourished nursing infants. Prostaglandins Leukot Essent Fatty Acids 63(6):385-90

42. Heird WC and Lapillonne A (2005). The role of essential fatty acids in development. Annu. Rev. Nutr. 25:549-71

43. Birch E, Hoffman D, Uauy R, Birch G. y Prestidge C (1998). Visual acuity and the essentiality of docosahexaenoic acid and arachidonic acid in the diet of term infants. Pediatr. Res.44: 201-209.

44. Hoffman D, Birch E, Birch D, Uauy R, Castañeda Y, Lapus G and Wheaton D (2000). Impact of early dietary intake and blood lipid composition of long-chain polyunsaturated fatty acids on later visual development. JPGN 31: 540-543

45. Agostoni C (2008). Role of Long-chain Polyunsaturated Fatty Acids in the First Year of Life. JPGN 47 S41-S44

46. Sala-Vila A, Castellote A, Campoy C, Rivero M, Rodríguez-Palmero M y López-Sabater MC (2004). The Source of Long-Chain PUFA in Formula Supplements Does Not Affect the Fatty Acid Composition of Plasma Lipids in Full-Term Infants. J. Nutr. 134: 868-873,

47. Qi B, Fraser T, Mugdorf S, Dobson G, Sayanova O, Butler J, Napier J.A, Stobart A.K and Lazarus C.M. (2004). Production of very long chain polyunsaturated omega-3 and omega6 fatty acids in plants Nature Biotechnol 22, 739-745

48. Napier J (2007). Transgenic plants as a source of fish oils: healthy, sustainable and GM. J Sci Food Agric 87:8-12

49. Kang J.X, Wang J, Wu L, and Kang Z.B (2004). Transgenic mice: Fat-1 mice convert w-6 to w-3 fatty acids. Kang J.X, Wang J, Wu L, and Kang Z.B Nature 427698

ISSN 1695-6141

๑ COPYRIGHT Servicio de Publicaciones - Universidad de Murcia 